\title{
Analoge Berechnung moderner Verfahren
}

\author{
Die Entstehung einer Karies verläuft bekanntlich nicht kontinuierlich und progredient, vielmehr \\ ist sie - abhängig von unterschiedlichsten Faktoren - in der Regel geprägt von wechselnden \\ Phasen der De- und Remineralisation. So kann sich Karies in unterschiedlich starken Ausprägun- \\ gen und Aktivitäten darstellen. Die richtige Einschätzung der kariösen Veränderung bezüglich \\ ihres Schweregrades bestimmt die Therapieentscheidung.
}

Für die routinemäßige Feststellung von kariösen Läsionen haben sich im täglichen Behandlungsablauf unterschiedliche Methoden etabliert. Die Kariesdiagnose erfolgt traditionell in erster Linie durch die direkte Sichtkontrolle (visuelle Inspektion) und die zahnärztliche Sonde [im Rahmen der eingehenden zahnärztlichen klinischen Untersuchung nach der Gebührenordnung für Zahnärzte (GOZ) Nr. 0010] ohne Zuhilfenahme weiterer diagnostischer Geräte sowie durch die radiologische Diagnostik wie Bissflügelaufnahmen oder andere Röntgenaufnahmen [vgl. Gebührenordnung für Ärzte (GOÄ) Nrn. 5000, 5004].

\section{Weitere Verfahren ohne GOZ-Nummer}

Neben diesen klassischen Verfahren der Kariesdiagnostik haben sich in neuerer Zeit aber weitere Untersuchungsmethoden etabliert, die von der GOZ 2012 nicht berücksichtigt werden. Zur äußerlichen Untersuchung von Zähnen vor einer Intervention sind verschiedene technisch anspruchsvolle Untersuchungsverfahren verfügbar (Kariesprimärdiagnostik zur Unterstützung der Entscheidung hinsichtlich eines invasiven oder eines eher abwartenden Therapieansatzes). Diese beruhen auf unterschiedlichen optischen Nachweisverfahren, unter anderem Laserdioden, Fluoreszenz oder Transillumination.

Diese apparativen, jedoch Röntgenstrahlen vermeidenden diagnostischen Verfahren sind entsprechend ihrem selbstständigen Charakter - ebenso wie die Röntgentechnik - gesondert berechnungsfähig. Sie bringen einen spezifischen Zusatznutzen bei der Kariesdiagnostik und der dazugehörigen Therapieentscheidung und haben neben dieser eindeutigen medizinischen Indikation noch den weiteren Vorteil der Reduktion der Röntgenbelastung für den Patienten, insbesondere bei einer engmaschigen Kariesverlaufskontrolle.

\section{Grenze zwischen Prophylaxe und Therapie}

Die GOZ 2012 berücksichtigt in einigen Neuformulierungen von Leistungslegenden eine moderne wissenschaftliche, sehr differenzierte Betrachtung der Frage, wann insbesondere Initialkaries klassisch mittels Füllungstechnik behandelt werden muss und wann (noch) nicht. So wird zum Beispiel in der Leistungslegende der GOZ-Nr. 1020 die Fluoridierung zur Kariestherapie („Lokale Fluoridierung zur Verbesserung der Zahnhartsubstanz, zur Kariesvorbeugung und -behandlung mit Lack oder Gel“) explizit erwähnt.

Derartige Aufweichungen der klassischen Grenze zwischen Prophylaxe und Therapie ergeben sich erst unter der Berücksichtigung der Tatsache, dass entsprechend hochtechnisierte, neue Diagnostikverfahren zur Verfügung stehen, die eine so differenzierte Entscheidung zwischen nichtinvasiver und invasiver
Therapie ermöglichen. Die Gebührenordnung nimmt damit indirekt Kenntnis von diesen neuen Diagnostikverfahren. Leider wurden aber entsprechende Gebührenziffern für eine solche notwendige apparative Diagnostik nicht aufgenommen. Insofern ist es richtig, diese entsprechend $₫ 6$ Abs. $1 \mathrm{GOZ}$ analog zu berechnen.

Zudem können während der Kariesentfernung spezielle Kariesdetektoren zur Überprüfung noch vorhandener Karies beziehungsweise der Kariesfreiheit dienen. Hierfür wurde schon vor längerer Zeit das chemische Verfahren zur Detektion größerer Hohlräume eingeführt. Bei der chemischen Hohlraumdetektion werden durch den Farbstoff gegebenenfalls noch vorhandene kariöse, demineralisierte Bezirke, die größere Poren als gesundes Dentin aufweisen, identifiziert.

Derzeit werden hierfür neue Systeme entwickelt, die auf Redox-Reaktionen oder kovalenten Bindungen an denaturiertem Kollagen beruhen. Schon praxisreif ist eine physikalische Alternative, die fluoreszenzunterstützte Kariesexkavation, die allerdings wie die oben genannten Systeme an die Anschaffung und Unterhaltung spezieller technischer Instrumente gebunden ist.

\section{Diagnostische Verfahren analog berechnen}

Die Anwendung auch dieser zusätzlichen diagnostischen Verfahren stellt eine jeweils selbstständige zahnärztliche Maßnahme dar, die - da sie nicht in der GOZ 2012 enthalten sind - ana$\log$ nach $\$ 6$ Abs. 1 GOZ zu berechnen sind. Die geeigneten Analogpositionen finden sich unter Berücksichtigung des tatsächlich gegebenen Kosten- und Zeitaufwandes und sollen hinsichtlich ihrer Art mit der tatsächlichen Leistung verwandt sein. Beim Kostenaufwand sind gerätespezifische Besonderheiten und der notwendige Einsatz von Einmalmaterial vom Zahnarzt mit zu berücksichtigen.

Die Ausführungen beruhen auf dem GOZ-Kommentar von Liebold/Raff/Wissing.

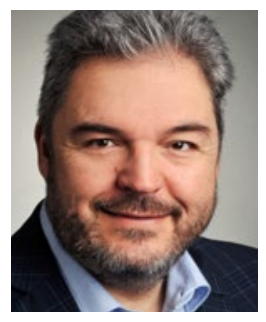

Dr. Dr. Alexander Raff

Mitglied im GOZ-Expertenrat des FVDZ 